\title{
A New Type of Waveguide Distribution for the Accelerator Module Test Facility of the European XFEL
}

\author{
V. Katalev ${ }^{1}$, S. Choroba ${ }^{1}$, A. Seliverstov ${ }^{2}$ and E. Apostolov ${ }^{3}$ \\ 1. Deutsches Elektronen-Synchrotron, DESY, MHF-p, Hamburg 22607, Germany \\ 2. Scientific \& Production Company FERRITE Ltd., St. Petersburg 196084, Russia \\ 3. MicroPlus-Apostolov Ltd., Sofia 1505, Bulgaria
}

\begin{abstract}
The European XFEL, which has been constructed at DESY in Hamburg, Germany, is an X-ray-Free Electron Laser, which provides X-ray light of unprecedented properties for different experiments in physics, chemistry, biology and technology [1]. The XFEL is based on superconducting cavity technology, which is required to accelerate an electron beam up to $17.5 \mathrm{GeV}$. The facility is installed about $20 \mathrm{~m}$ underground in a $3.4 \mathrm{~km}$ long tunnel of $5.2 \mathrm{~m}$ diameter. High power RF systems are required to accelerate the beam to the required energy. Each RF station provides RF power to 4 accelerator modules with 8 superconducting cavities by a waveguide RF distribution system [2, 3]. Besides electrical and RF properties, mechanical properties are of high importance, since the waveguide distribution system and its components have to be manufactured, assembled and aligned with high precision. In order to test 100 superconducting accelerator modules within two years three test benches have been created in the AMTF (accelerator module test facility) to achieve the rate of one superconducting module per week. Each RF station of the test facility consists of a 5 MW RF station at $1.3 \mathrm{GHz}, 1.37 \mathrm{~ms}$ pulse width and $10 \mathrm{~Hz}$ repetition rate, with a waveguide distribution system. Each waveguide distribution supplies RF power to eight cavities, four times a pair of cavities. The distribution allows for a maximum power of 1 MW per cavity when the distribution is switched to a mode supplying power to only four cavities. A new type of $1 \mathrm{MW}$ isolator and a new compact $5 \mathrm{MW}$ power divider have been developed to achieve that goal. We present the waveguide distribution for this test stand and describe the performance of the different elements.
\end{abstract}

Key words: High power RF, superconducting RF, particle accelerator, kinematic design, precision mechanic adjustment, exact constraint design.

\section{Requirements for the AMTF Waveguide Distribution}

The AMTF (accelerator module test facility) waveguide distribution should meet several specifics sometimes conflicting requirements. On one side the distribution should have a compact size since there is only limited space in the AMTF shielding tunnel. On the other side it should supply high pulse RF power to the individual cryomodule cavities with high flexibility by only one power klystron. In addition the waveguide distribution has to protect the klystron against reflected power from superconducting cavities.

The three basic requirements are:

Corresponding author: Dr. V. Katalev, senior scientist, research fields: High Power RF,RF distribution, particle accelerators.
- Power per cavity:

$1 \mathrm{MW}$ max pulse power

$2.2 \mathrm{~kW}$ max average power

- One 5 MW klystron as RF power source

- The waveguide distribution layout must allow for a free access to the cavity couplers for local clean room installation.

In order to satisfy these conditions specific waveguide components, such as a $1 \mathrm{MW}$ isolator and a $5 \mathrm{MW}$ power divider have been developed and integrated in the waveguide distribution. A 3D-view of the AMTF waveguide distribution is shown in Fig. 1.

During maintenance of RF station the klystron has to be connected to two dummy loads. Therefore a mechanical waveguide switch is installed which allows for quick connection of the klystron and the loads. 


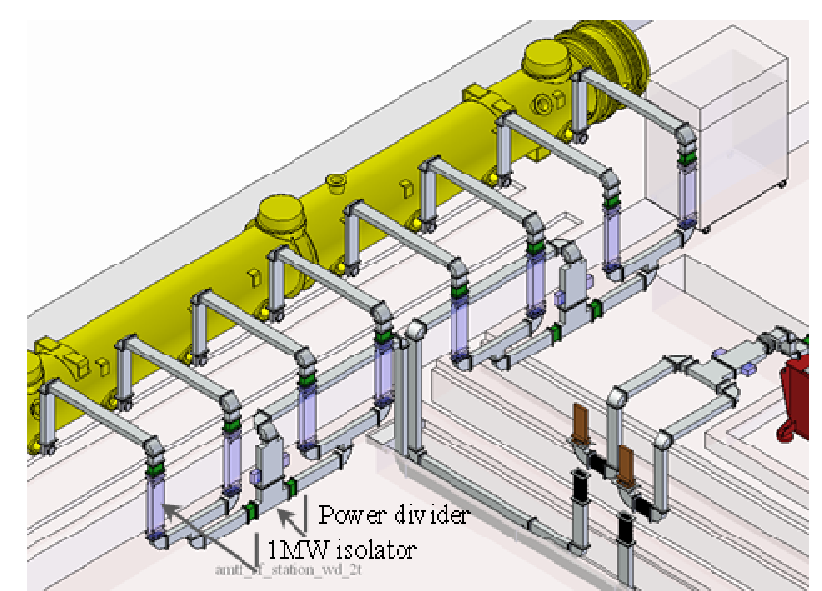

Fig. 1 3D-view of the AMTF waveguide distribution.

\section{1 MW Isolator}

In order to protect the klystrons in the XFEL tunnel against reflected power the $\mathrm{Y}$-junction circulators with matched and four port phase shift circulators with two matched loads are used. For the AMTF waveguide distribution the isolators could not be used since the Y-junction circulators are limited in power and phase shift circulators are too big. Therefore a new type of a 1 MW isolator has been developed by the Company "FERRITE" from St. Petersburg, Russia. The new FWHI3-27A type isolator uses the nonreciprocal energy absorption in the ferrite elements by ferromagnetic resonance for waves with circular polarization [4]. An overview of the device (without magnetic system) is shown in Fig. 2.

In this device the reflected power is absorbed in the ferrite material itself and not in an external matched load. The special type of the ferrite, its shape and location as well as the design of water cooling system allow an increase of the average power. The magnet system uses a high-performance permanent Fe-Nd-B magnet. To decrease the weight and size of the device the waveguide height has been reduced by three times compared with a standard WR650. But due to a specific configuration and tuning of the magnetic system the power capability of device has been saved.

After test of the first prototype at DESY and further improvement more than 30 isolators have been produced meeting the following specifications:

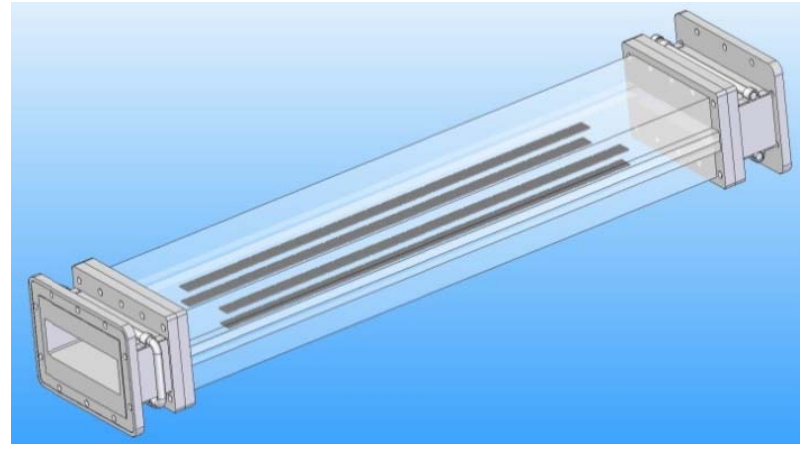

Fig. 2 View of the S.P.A. FERRITE 1 MW isolator.

- Pulse power, max $1 \mathrm{MW}$

- Average power, max $2.2 \mathrm{~kW}$

- Input SWR, max 1.15 (at full reflection)

- Isolation, min $30 \mathrm{~dB}$

- Insertion loss, max $0.5 \mathrm{~dB}$

- Full reflection any phase

- Length $950 \mathrm{~mm}$

- Height 1/3 of WR650

- Flange UDR-14D

All isolators have been successfully tested up to 1.15 MW input power.

\section{5 MW Compact Power Divider}

The 5 MW power divider has been developed in cooperation with MicroPlus-Apostolov Company, Sofia, Bulgaria. The device is designed in accordance with a classical scheme: shunt tee, two phase shifters and hybrid with integrated $\mathrm{H}$ bends. Each waveguide component has been designed especially for this power divider. Simulation results of the device with CST MWS (Microwave Studio) are shown in Fig. 3.

Depending on the phase shifters position the RF power is distributed between the two outputs ports. The integrated phase shifter is similar to the phase shifter for waveguide distribution in the XFEL tunnel [5].

The mechanical construction of the phase shifters is given in Fig. 4. A piston 7 is driven by a linear positioning system. The system consists of a stepping motor 1 integrated with driving screw 12 and nut 13 without backlash. By flexural kinematic coupling 4 with the details 5, 6 and 14 the piston 7 are connected to a moving carriage 2 on a linear guide 8 . The flexural 


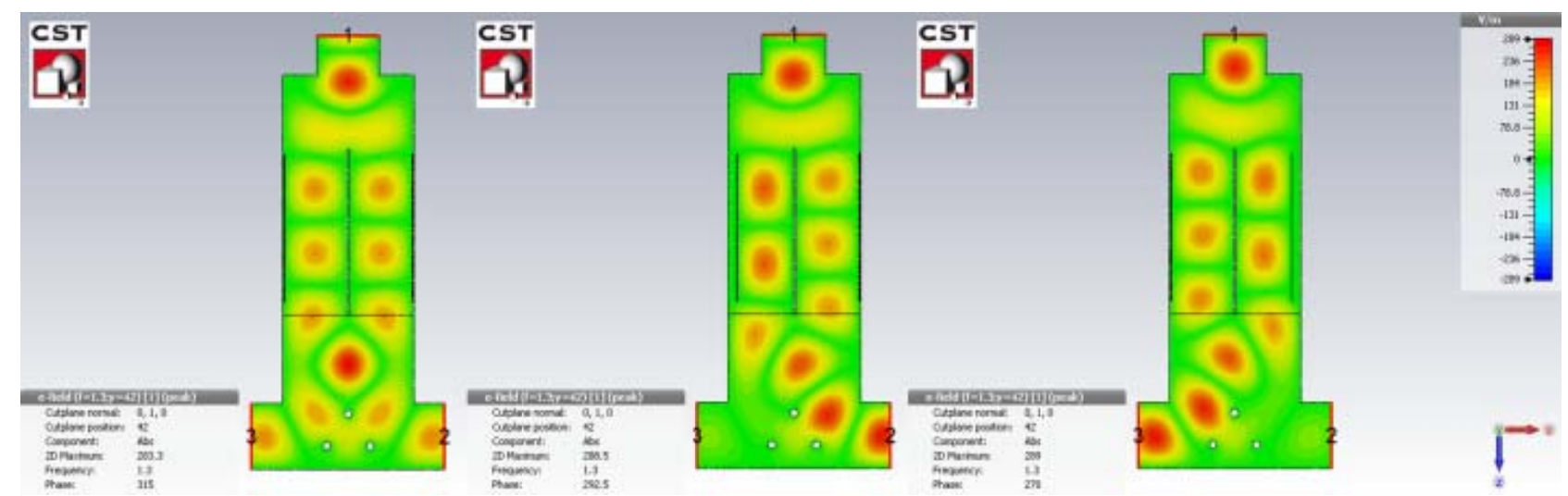

Fig. 3 RF power distribution depending on the phase shifter piston position.

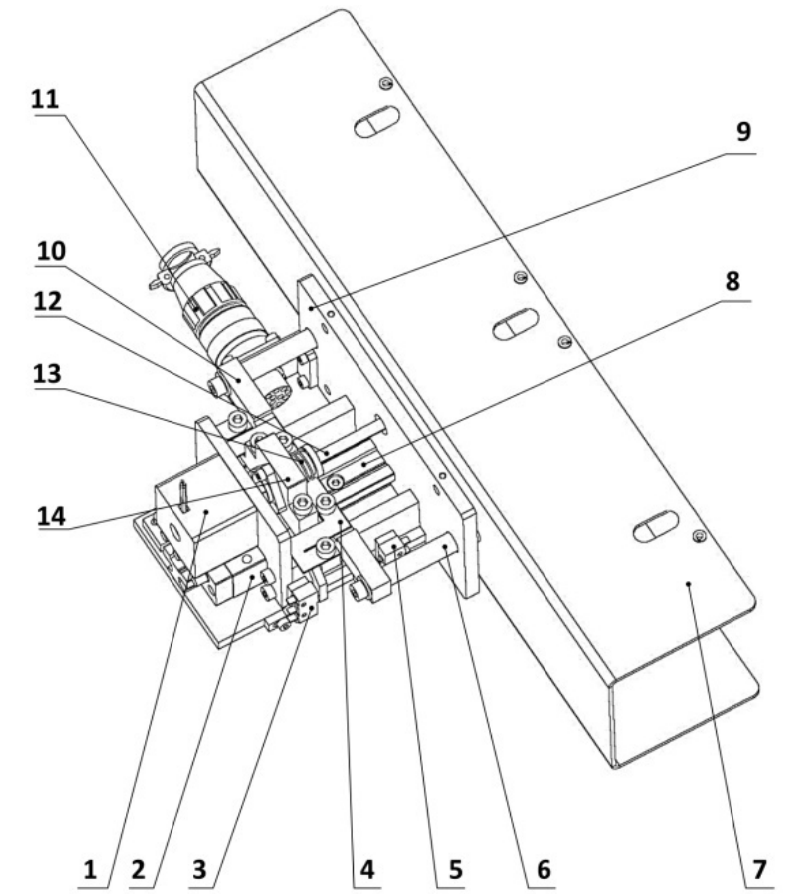

Fig. 4 Mechanical construction of the phase shifter.

kinematic coupling provides four degrees of freedom and two constraints for the piston. Housing 9 supports all fixed components of the phase shifter, including home position sensor 3, limit switch 5 and electrical connector 11.

The piston range is $30 \mathrm{~mm}$ and the linear positional accuracy is better than $0.1 \mathrm{~mm}$. The phase shifter has a compact construction with the possibility to operate with gas overpressure in the waveguide (the seals are not included in Fig. 4). To reduce impact of mounting errors and thermal deformation during operation the design of the phase shifter thoroughly meets the principle of the kinematic design [6, 7].

To decrease the size of the power divider a new type of hybrid junction with integrated bends has been also developed. It allows reducing the device length of the $5 \mathrm{MW}$ divider down to $700 \mathrm{~mm}$.

The $5 \mathrm{MW}$ power divider parameters are:

- Pulse power, max $5 \mathrm{MW}$

- Average power, max $50 \mathrm{~kW}$

- Input SWR, max 1.2

- Isolation, min $27 \mathrm{~dB}$

- Length $700 \mathrm{~mm}$

- Width $550 \mathrm{~mm}$

- Flange UDR-14D

- Overpressure, max 0.5 bar.

The combination of these power dividers allows distributing the RF power from the $5 \mathrm{MW}$ klystron with high flexibility to any pair of the cavities with a maximum power up to $1 \mathrm{MW}$ for each cavity.

\section{Waveguide Distribution for AMTF RF Station}

The AMTF contains 3 RF test stands to test cryomodules for the XFEL. This allows a test rate of one cryomodule per week. Each RF station consists of a 5 MW RF station and the specific waveguide distribution which supplies a cryomodule. The schematic of the waveguide distributing system is shown in Fig. 5.

Each pair of cavities is supplied through a shunt tee which divides the RF power equally to the two cavities. 


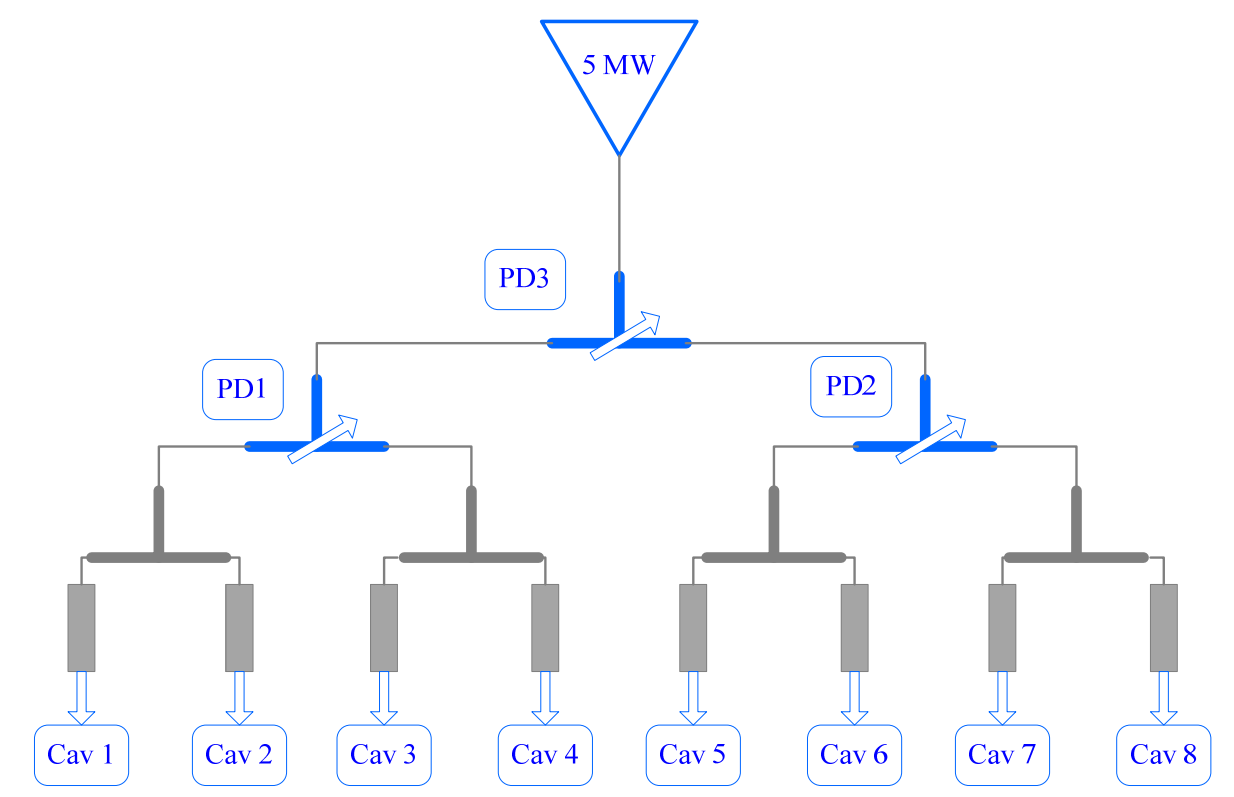

Fig. 5 Schematic of the power distribution system.

Table 1 Power distribution possibilities for the cavities at AMTF RF station.

\begin{tabular}{llllllllll}
\hline N & $\begin{array}{l}\text { P } \\
\text { kly, }\end{array}$ & \multicolumn{7}{c}{$\mathrm{P}_{\text {cavity }}, \mathrm{MW}$} \\
\cline { 2 - 10 } & $\mathrm{MW}$ & 1 & 2 & 3 & 4 & 5 & 6 & 7 & 8 \\
\hline 1 & 4.5 & 0.5 & 0.5 & 0.5 & 0.5 & 0.5 & 0.5 & 0.5 & 0.5 \\
2 & 4.5 & 1 & 1 & 1 & 1 & 0 & 0 & 0 & 0 \\
3 & 4.5 & 1 & 1 & 0 & 0 & 1 & 1 & 0 & 0 \\
4 & 4.5 & 1 & 1 & 0 & 0 & 0 & 0 & 1 & 1 \\
5 & 4.5 & 0 & 0 & 1 & 1 & 1 & 1 & 0 & 0 \\
6 & 4.5 & 0 & 0 & 1 & 1 & 0 & 0 & 1 & 1 \\
7 & 4.5 & 0 & 0 & 0 & 0 & 1 & 1 & 1 & 1 \\
8 & 2.2 & 1 & 1 & 0 & 0 & 0 & 0 & 0 & 0 \\
9 & 2.2 & 0 & 0 & 1 & 1 & 0 & 0 & 0 & 0 \\
10 & 2.2 & 0 & 0 & 0 & 0 & 1 & 1 & 0 & 0 \\
11 & 2.2 & 0 & 0 & 0 & 0 & 0 & 0 & 1 & 1 \\
\hline
\end{tabular}

For two pairs of cavities a $5 \mathrm{MW}$ power divider is installed. It allows distribution of RF power between two pairs. Another $5 \mathrm{MW}$ power divider which is installed after the klystron distributes RF power between two halves of the cryomodule.

Table 1 summarizes typical operation conditions of the AMTF waveguide distribution. The power generated by the klystrons is more than the sum of the power supplied to the cavities since losses in the distribution have to be taken into account. $1 \mathrm{MW}$ is the maximum pulse power which can be accepted by one cavity and its input coupler.
The waveguide distribution contains 15 directional couplers with high directivity (more than $40 \mathrm{~dB}$ ) to measure and control the distribution of RF power.

For RF station conditioning, two manual RF switches are provided. The RF switch allows for connection of the klystron to the dummy loads. The RF switch consists of a standardized H-bend and a flexible waveguide that allows to avoid electrical field breakdown and to compensate for mechanical deviations of waveguide components. The RF switch has contacts for a personnel interlock.

The waveguide distribution with support frame in the AMTF shielding tunnel is shown in Fig. 6.

The two basic components of the waveguide distribution in AMTF shielding tunnel, one $5 \mathrm{MW}$ power divider and one $1 \mathrm{MW}$ isolator are shown in Fig. 7. The waveguide components and the entire distribution have been successfully tested up to full RF power of $5 \mathrm{MW}$.

The design of the distribution system fully complies with the principles of kinematic design in order to avoid any mechanical stress during installation and stress due to thermal expansion during operation [8]. As an example the mechanical connection of the isolator to the supporting structure is shown in 


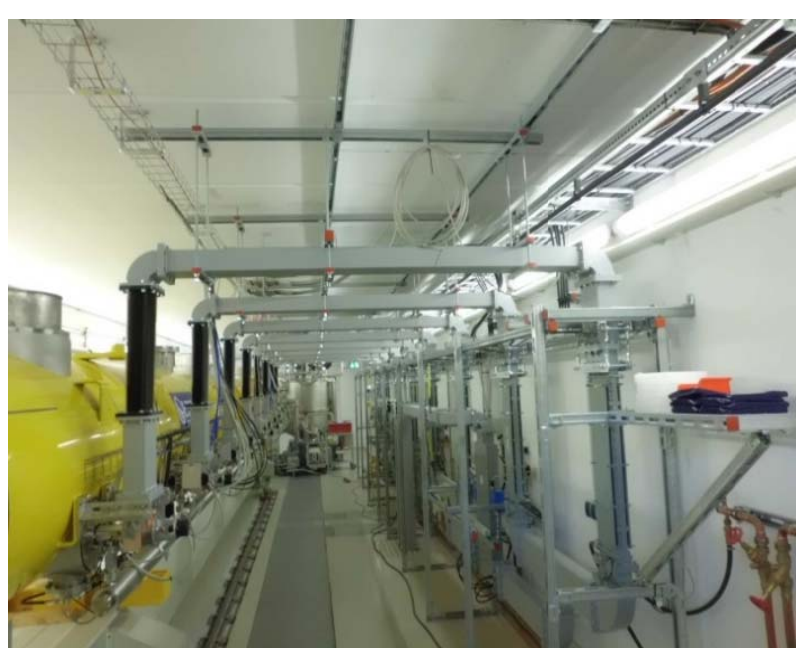

Fig. 6 Waveguide distribution connected to a cryomodule in the AMTF shielding tunnel.

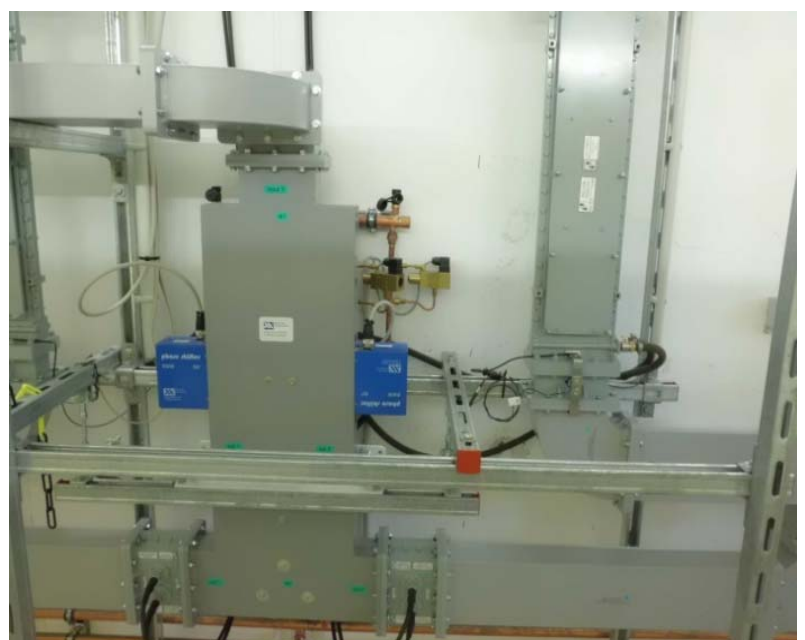

Fig. $75 \mathrm{MW}$ power divider and $1 \mathrm{MW}$ isolator in the AMTF shielding tunnel.

Fig. 8. Isolator frame 2 consists of brackets and bolted joints. The frame is hanging on three bars 1 . Each bar of adjustable length has two spherical joints. With these three bars it is possible to provide precise adjustment of the isolator position along the " $z$ " axis and rotation around " $\mathrm{x}$ " and " $\mathrm{y}$ ". Bar 3 is used for isolator positioning. Bar 3 also determines the stiffness of isolator supporting axis “ $y$ ”. The three vertical bars 1 and bar 3 provide two degrees of freedom for the isolator in total (translation along axis " $\mathrm{x}$ " and rotation around the axis " $\mathrm{z}$ "). All other movement is constrained.

Power dividers are connected to the supporting structure by the same kinematic design described.
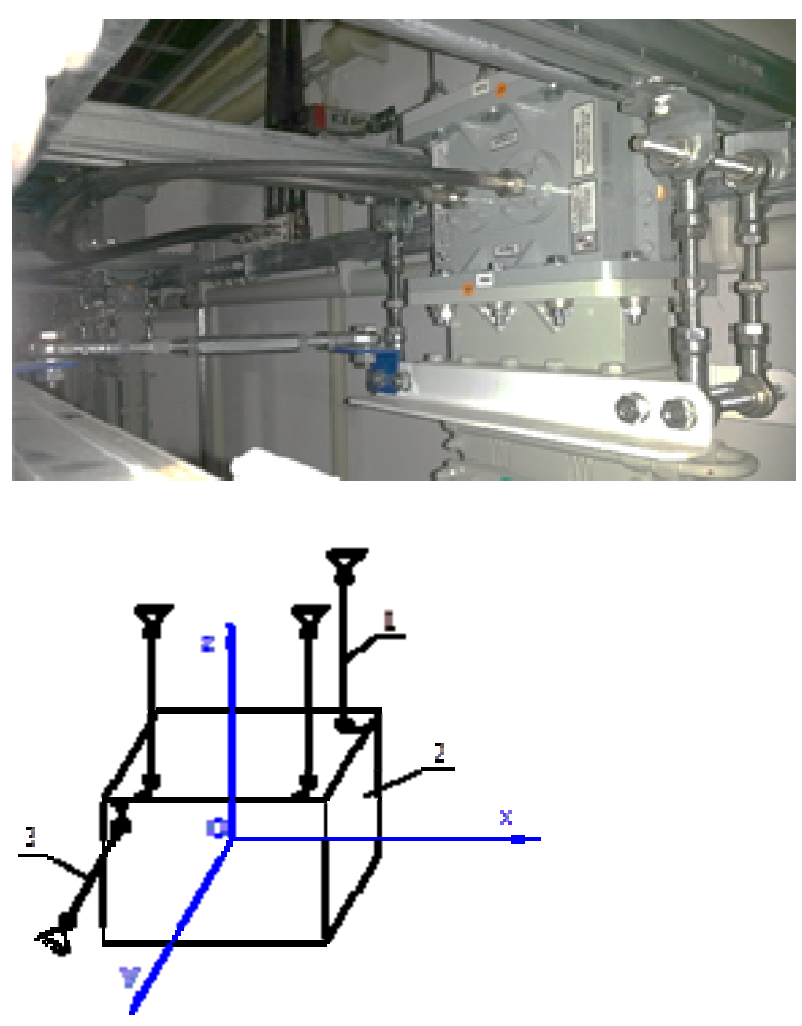

Fig. 8 Isolator support and its mechanical kinematic equivalent.

\section{Conclusions}

Three RF stations have been successfully installed at AMTF. The waveguide distribution system for each station has been tuned and tested up to full power. Since the end of 2013 all accelerator modules for the European XFEL have been tested, prepared at the AMFT and finally installed in the XFEL tunnel.

\section{Acknowledgement}

The authors are grateful to all the members of teams of DESY MHF-p, FERRITE and Micro Plus contribution to successful installation, tuning and commissioning of the AMTF waveguide distributions.

All figures (c) 2017 by the authors licensed under CC-BY 4.0.

\section{References}

[1] “The European X-Ray Free-Electron Laser." TDR, DESY 2006-097.

[2] Choroba, S. 2007. "Design and Status of the XFEL RF System.” In Proceedings of the PAC07, Albuquerque, 
New Mexico.

[3] Katalev, V., and Choroba, S. 2007. "Compact Waveguide Distribution with Asymmetric Shunt Tees for the European XFEL.” In Proceedings of PAC07, Albuquerque, New Mexico, USA.

[4] Harvey, A. F. 1963. Microwave Engineering. London and New York: Academic Press.

[5] Katalev, V., and Choroba, S. 2006. "Waveguide
Distribution Systems for the European XFEL.” In Proceedings of EPAC 2006, Edinburgh, Scotland.

[6] Slocum, A. H. 1992. Precision Machine Design. Society of Manufacturing, ISBN-13: 978-0872634923.

[7] Blanding, D. L. 1999. Exact Constraint: Machine Design Using Kinematic Principles. ASME Press.

[8] Hale, L. C. 1999. "Principles and Techniques for Designing Precision Machines.” UCRL-LR-133066. 